TRANSACTIONS OF THE

AMERICAN MATHEMATICAL SOCIETY

Volume 357, Number 1, Pages 31-44

S 0002-9947(04)03682-7

Article electronically published on August 19, 2004

\title{
SYMMETRICALLY APPROXIMATELY CONTINUOUS FUNCTIONS, CONSISTENT DENSITY THEOREMS, AND FUBINI TYPE INEQUALITIES
}

\author{
P. D. HUMKE AND M. LACZKOVICH
}

\begin{abstract}
Using the continuum hypothesis, Sierpiński constructed a nonmeasurable function $f$ such that $\{h: f(x+h) \neq f(x-h)\}$ is countable for every $x$. Clearly, such a function is symmetrically approximately continuous everywhere.

Here we to show that Sierpiński's example cannot be constructed in ZFC. Moreover, we show it is consistent with ZFC that if a function is symmetrically approximately continuous almost everywhere, then it is measurable.
\end{abstract}

\section{INTRODUCTION}

The problem we address in this paper concerns the measurability of symmetrically approximately continuous functions.

It is well known that every approximately continuous function is measurable; in fact, a function is measurable if and only if it is approximately continuous almost everywhere.

A real function $f$ is called symmetrically continuous at the point $x$ if

$$
\lim _{h \rightarrow 0}[f(x+h)-f(x-h)]=0 .
$$

It was proved by I. N. Pesin [9] and, independently, by D. Preiss [10] (see also [12, Theorem 2.26]) that if the function $f$ is symmetrically continuous everywhere, then $f$ is measurable (even continuous almost everywhere). This result was improved by J. Uher [13] who showed that if $f$ is symmetrically continuous almost everywhere, then $f$ is necessarily continuous almost everywhere (and hence, measurable).

We call the function $f$ symmetrically approximately continuous at the point $x$ if $\operatorname{lim~app}_{h \rightarrow 0}[f(x+h)-f(x-h)]=0$; that is, if there exists a measurable set $H$ having a density point at zero such that $\lim _{h \rightarrow 0, h \in H}[f(x+h)-f(x-h)]=0$. Now the results above would suggest that if a function $f$ is symmetrically approximately continuous everywhere (or even almost everywhere), then $f$ is necessarily measurable. However, as Sierpiński showed in [11], this is not the case. Using the continuum hypothesis, Sierpiński constructed a nonmeasurable function $f$ such that $\{h: f(x+h) \neq f(x-h)\}$ is countable for every $x$. Clearly, such a function is

Received by the editors March 10, 2003.

2000 Mathematics Subject Classification. Primary 03E35; Secondary 28A20, 26A03.

Key words and phrases. Fubini, symmetrically approximately continuous, covering number, shrinking number.

The second author's research was supported by the Hungarian National Foundation for Scientific Research Grant No. T032042. 
symmetrically approximately continuous (even symmetrically approximately differentiable) everywhere. (For related results and problems also involving symmetric approximate differentiability, see [1] and [12].)

Our aim is to show that Sierpiński's example cannot be constructed in ZFC. Moreover, it is consistent with ZFC that if a function is symmetrically approximately continuous almost everywhere, then it is measurable. What we actually prove is that the inequality $\operatorname{shr}(\mathcal{N})<\operatorname{cov}(\mathcal{N})$ implies our statement (Theorem 12). Then the consistency result follows from the consistency of $\operatorname{shr}(\mathcal{N})<\operatorname{cov}(\mathcal{N})$, which is well known (see [8] or [6]).

Every argument proving the continuity or measurability of a symmetrically (approximately) continuous function $f$ must show that there is a large set on which the oscillation of $f$ is small. Usually this is done as follows: we fix an $\varepsilon>0$ and define $S=\{(x, x+h):|f(x+h)-f(x-h)|<\varepsilon\}$. If $f$ is symmetrically continuous at $x$, then $S_{x}=\{y:(x, y) \in S\}$ is a neighbourhood of $x$; if $f$ is symmetrically approximately continuous at $x$, then $S_{x}$ has density 1 at $x$. Then we show that there is a large set $E$ such that any two points $x, y \in E$ can be joined by a $k$-chain in the following sense: there are points $x=x_{0}, x_{1}, \ldots, x_{k}=y$ and $p_{1}, \ldots, p_{k}$ such that $x_{i-1}+x_{i}=2 p_{i}$ and $\left(p_{i}, x_{i-1}\right) \in S$ for every $i=1, \ldots, k$. It is clear that the existence of these $k$-chains implies that $|f(x)-f(y)|<k \cdot \varepsilon$ for every $x, y \in E$, and thus the oscillation of $f$ on $E$ is at most $2 k \cdot \varepsilon$. Each of the papers 9, [10, 13, and [5] contains an argument of this kind. (See also [12], where these arguments are discussed as 'covering theorems'.) Our proof also follows this line: we show, under $\operatorname{shr}(\mathcal{N})<\operatorname{cov}(\mathcal{N})$, that if $S_{x}$ has density 1 for almost every $x$, then there is a measurable set $F$ of positive measure such that every two points of $F$ can be joined by an 8-chain (Theorem [11). A similar but weaker statement was proved by C. Freiling in a more general setting in [3] 1 . Freiling's proof shows, also under $\operatorname{shr}(\mathcal{N})<\operatorname{cov}(\mathcal{N})$, that if $S_{x}$ has density 1 for every $x$, then there is an interval $I$ such that every two points of $I$ can be joined by a finite chain. However, his proof yields no uniform bound on the lengths of the chains.

One of the tools of our proof is a consistent inequality between some iterated integrals of bounded functions of two variables. Since these results might have some interest in themselves, we separated them in Section 3.

\section{Notation}

We denote by $\mathcal{N}$ the ideal of null sets in $\mathbb{R}$. We shall need the following classical cardinal numbers connected to $\mathcal{N}$.

$\operatorname{non}(\mathcal{N})=\min \{|A|: A \subset \mathbb{R}, A \notin \mathcal{N}\}$

$\operatorname{cov}(\mathcal{N})=\min \{|\mathcal{F}|: \mathcal{F} \subset \mathcal{N} \& \bigcup \mathcal{F}=\mathbb{R}\}$

$\operatorname{shr}(\mathcal{N})=\min \{\kappa: \forall A \subset \mathbb{R}(A \notin \mathcal{N} \Longrightarrow \exists B \subset A,|B| \leq \kappa, B \notin \mathcal{N})\}$.

In other words, $\operatorname{shr}(\mathcal{N})$ is the smallest cardinal $\kappa$ such that any subset $A$ of $\mathbb{R}$ not belonging to $\mathcal{N}$ contains a subset $B$ such that $|B| \leq \kappa$ and $B \notin \mathcal{N}$.

The Lebesgue outer measure is denoted by $\lambda^{*}$. The Lebesgue inner measure of a set $A \subset \mathbb{R}$ is defined as $\lambda_{*}(A)=\max \left\{\lambda^{*}(H): H \subset A\right.$ is measurable $\}$. As usual, $\lambda(A)$ means the measure of the Lebesgue measurable set, $A$.

\footnotetext{
${ }^{1}$ Freiling credits the techniques used to prove his theorem to Erdős, McGrotty, and Sierpiński. See $[3$ for details.
} 
The upper and lower outer density of a set $A \subset \mathbb{R}$ at the point $x$ are defined by

$$
\bar{d}^{*}(A, x)=\limsup _{h \rightarrow 0+} \lambda^{*}(A \cap[x-h, x+h]) /(2 h)
$$

and

$$
\underline{d}^{*}(A, x)=\liminf _{h \rightarrow 0+} \lambda^{*}(A \cap[x-h, x+h]) /(2 h) .
$$

If, in these definitions, the Lebesgue outer measure $\lambda^{*}$ is replaced by the Lebesgue inner measure $\lambda_{*}$, then we obtain the upper and lower inner density of $A \subset \mathbb{R}$, denoted by $\bar{d}_{*}(A, x)$ and $\underline{d}_{*}(A, x)$. If $\bar{d}^{*}(A, x)=\underline{d}^{*}(A, x)$, then we say that $A$ has outer density at $x$, and define $d^{*}(A, x)$ as the common value of $\bar{d}^{*}(A, x)$ and $\underline{d}^{*}(A, x)$. We say that $x$ is an outer density point of $A$ if $d^{*}(A, x)=1$. If $\bar{d}_{*}(A, x)=$ $\underline{d}_{*}(A, x)$ holds, then we say that $A$ has inner density at $x$, and define $d_{*}(A, x)$ as the common value of $\bar{d}_{*}(A, x)$ and $\underline{d}_{*}(A, x)$. If $d_{*}(A, x)=1$, then we say that $x$ is a density point of $A$.

If $(X, \Sigma, \mu)$ is a measure space, we shall denote by $\mu^{*}$ the outer measure generated by $\mu$. That is, for each $H \subset X, \mu^{*}(H)=\inf \{\mu(A): H \subset A, A \in \Sigma\}$.

Let $A \subset X$ be measurable. If $f: A \rightarrow \overline{\mathbb{R}}$, then we shall denote by $\int_{A} f d \mu(x)$ the supremum of the integrals $\int_{A} g d \mu(x)$, where $g: A \rightarrow \overline{\mathbb{R}}$ is an arbitrary summable function such that $g \leq f$ everywhere on $A$. If there is no such $g$, then we put $\underline{\int}_{A} f d \mu(x)=-\infty$. The upper integral $\bar{\int}_{A} f d \mu(x)$ is defined analogously.

The interval $[0,1]$ will be denoted by $I$. When integrating over $I$, we shall omit the subscript $I$; that is, $\underline{\int}, \bar{\int}, \int$ stand for $\underline{\int}_{I}, \bar{\int}_{I}, \int_{I}$.

Let $\Omega$ denote the measure space $I^{\mathbb{N}}=I \times I \times \ldots$ with the product measure $\nu$. The generic element of $\Omega$ will be denoted by $\bar{y}=\left(y_{1}, y_{2}, \ldots\right)$, where each $y_{i}$ belongs to $I$. Note that the measure space $(\Omega, \nu)$ is isomorphic to $(I, \lambda)$.

The characteristic function of a set $H$ is denoted by $\chi_{H}$. If $H \subset X \times Y$, then the sections of $H$ are defined as $H_{x}=\{y \in Y:(x, y) \in H\}$ and $H^{y}=\{x \in X$ : $(x, y) \in H\}$ for every $x \in X$ and $y \in Y$.

A set $H \subset I \times I$ is a Sierpinski set if $\lambda\left(H_{x}\right)=1$ for every $x \in I$ and $\lambda\left(H^{y}\right)=0$ for every $y \in I$.

Finally, a set $H \subset I \times I$ is a weak Sierpiński set if $\lambda\left(H_{x}\right)=1$ for every $x \in I$ and

$$
\lambda^{*}\left(\left\{y \in I: \lambda\left(H^{y}\right)=0\right\}\right)>0 .
$$

\section{Consistent Fubini type inequalities}

By a Fubini type inequality we shall mean an inequality of the form

$$
\int_{1}\left(\int_{2} f(x, y) d y\right) d x \leq \int_{3}\left(\int_{4} f(x, y) d x\right) d y
$$

where each of the signs $\int_{i}(i=1, \ldots, 4)$ stands for either $\int$ or $\bar{\int}$. There are 16 inequalities of this form. In this section our aim is to show that some of them hold consistently for every bounded $f: I^{2} \rightarrow \mathbb{R}$. More precisely, we shall prove that the following statement is consistent with ZFC: For every bounded function 
$f:(I \times I) \rightarrow \mathbb{R}$ the following inequalities hold:

$$
\begin{aligned}
& \left\{\left(\underline{\left.\int f(x, y) d y\right) d x} \leq \bar{\int}\left(\bar{\int} f(x, y) d x\right) d y,\right.\right. \\
& \underline{\int\left(\int f(x, y) d y\right) d x} \leq \leq \underline{\int}\left(\bar{\int} f(x, y) d x\right) d y,
\end{aligned}
$$

and

$$
\bar{\int}\left(\underline{\int f}(x, y) d y\right) d x \leq \bar{\int}\left(\int f(x, y) d x\right) d y .
$$

Clearly, (2) is a consequence of each of (3) and (4). The consistency of (2) is closely related to a theorem of C. Freiling 2] stating that the following two statements are equivalent to each other:

(i)

$$
\int_{0}^{1}\left(\int_{0}^{1} f(x, y) d y\right) d x=\int_{0}^{1}\left(\int_{0}^{1} f(x, y) d x\right) d y
$$

holds for every bounded function $f: I^{2} \rightarrow \mathbb{R}$ for which the integrals involved in (5) exist;

(ii) there is no Sierpinski set.

Now the argument proving Freiling's theorem can be easily modified in order to prove that (ii) is also equivalent to the statement that (2) holds for every bounded $f: I^{2} \rightarrow \mathbb{R}$. Since the nonexistence of Sierpiński sets is consistent with ZFC (see, e.g., [7]), it follows that (2) holds consistently for every bounded $f$.

We remark that no Fubini type inequality can be proved in ZFC. Indeed, if $S$ is a Sierpiński set and $f=\chi_{S}$, then

$$
1=\int_{0}^{1}\left(\int_{0}^{1} f(x, y) d y\right) d x>\int_{0}^{1}\left(\int_{0}^{1} f(x, y) d x\right) d y=0,
$$

and thus

$$
\int_{1}\left(\int_{2} f(x, y) d y\right) d x>\int_{3}\left(\int_{4} f(x, y) d x\right) d y
$$

for every choice of $\int_{i}(i=1, \ldots, 4)$.

Before turning to the proof of the consistency of the stronger inequalities (3) and (4) we show that apart from (2), (3), and (4) there are no other consistent Fubini type inequalities.

It is well known that $[0,1]$ can be decomposed into continuum many pairwise disjoint sets of outer measure 1 . Let $[0,1]=\bigcup_{x \in[0,1]} H_{x}$ be such a decomposition, and put $H=\left\{(x, y) \in I^{2}: y \in H_{x}\right\}$ and $f=\chi_{H}$. Then $\lambda^{*}\left(H_{x}\right)=1$ for every $x \in I$ and $\lambda^{*}\left(H^{y}\right)=0(y \in I)$, as $H^{y}$ is a singleton for every $y \in I$. Thus $\bar{\int} f d x d y=0$ and $\int \bar{\int} f d y d x=1$. Consequently, the inequality $\int \bar{\int} f d y d x>\bar{\int} \int f d x d y$ holds for some bounded $f$ in ZFC. Then, a fortiori, neither of the following inequalities is true for every bounded $f$ :

$$
\underline{\iint} f d y d x \leq \int_{1} \int_{2} f d x d y, \quad \bar{\iint} f d y d x \leq \int_{1} \int_{2} f d x d y,
$$


for any choice of $\int_{1}$ and $\int_{2}$. This example shows that eight of the possible Fubini type inequalities are false in ZFC. Moreover, we can find other false inequalities by taking duals.

If a function $f$ satisfies (1), then the function $g(x, y)=-f(y, x)$ satisfies

$$
\int_{3}^{\prime}\left(\int_{4}^{\prime} g(x, y) d y\right) d x \leq \int_{1}^{\prime}\left(\int_{2}^{\prime} g(x, y) d x\right) d y
$$

where $\int_{i}^{\prime}=\bar{\int}$ if $\int_{i}=\underline{\int}$ and $\int_{i}^{\prime}=\underline{\int}$ if $\int_{i}=\bar{\int}$. (That is, (7) is obtained from (11) by switching its sides and by changing $\bar{\int}$ into $\int$ and $\int$ into $\bar{\int}$ in every occurrence.) Let us call (7) the dual of (11). (Thus (4) is the dual of (3).) Clearly, if (1) holds for every bounded $f$, then so does its dual. It is easy to check that the inequalities (6) together with their duals exclude all but four Fubini type inequalities that can be consistent with ZFC. The remaining inequalities are (2), (3), (4), and

$$
\bar{\int}\left(\underline{\int f}(x, y) d y\right) d x \leq \underline{\int\left(\int f(x, y) d x\right) d y .}
$$

We show that (8) is also false in ZFC. Let non $(\mathcal{N})=\kappa$, and let $A \subset I$ be a set such that $\lambda^{*}(A)>0$ and $|A|=\kappa$. Replacing $A$ by $I \cap \bigcup_{q \in \mathbb{Q}}(A+q)$ if necessary, we may assume that $\lambda^{*}(A)=1$. Let $A=\left\{x_{\alpha}: \alpha<\kappa\right\}$ be an enumeration of $A$, and put

$$
H=\left\{\left(x_{\alpha}, x_{\beta}\right): \alpha<\beta<\kappa\right\} \cup(A \times(I \backslash A)) .
$$

If $y=x_{\beta} \in A$, then $H^{y}=\left\{x_{\alpha}: \alpha<\beta\right\}$. Consequently, $\left|H^{y}\right|<\kappa$ and $\lambda^{*}\left(H^{y}\right)=0$ by the definition of $\kappa$. Since $\lambda^{*}(A)=1$, this implies $\int \bar{\int} \chi_{H} d x d y=0$. On the other hand, if $x=x_{\alpha} \in A$, then $I \backslash H_{x}=\left\{x_{\beta}: \beta \leq \bar{\alpha}\right\}$. Thus $\lambda^{*}\left(I \backslash H_{x}\right)=0$ and $\lambda_{*}\left(H_{x}\right)=1$ for every $x \in A$. Therefore $\iint \chi_{H} d y d x=1$ showing that (8) is not satisfied by $\chi_{H}$. Summing up: no Fubini type inequalities can be consistent with ZFC apart from (2), (3), and (4). The rest of this section will be devoted to the proof of consistency of these inequalities.

In the next three lemmas we assume that $(X, \Sigma, \mu)$ is a measure space, $A \subset X$ is measurable, and $f: A \rightarrow \overline{\mathbb{R}}$ is arbitrary.

Lemma 1. If $\int_{A} f d \mu(x)$ is finite, then there is a summable function $g: A \rightarrow \overline{\mathbb{R}}$ such that $g \leq f$ on $A, \int_{A} g d \mu(x)=\underline{\int}_{A} f d \mu(x)$, and for every $\varepsilon>0, \mu^{*}(\{x \in A$ : $f(x)<g(x)+\varepsilon\})=\mu(A)$.

Proof. Let $\int_{A} f d \mu(x)=a$, and choose summable functions $g_{n}$ such that $g_{n} \leq f$ and $\int_{A} g_{n} d \mu(x)>a-(1 / n)$. Put $g=\lim _{n} \max \left(g_{1}, \ldots, g_{n}\right)$. Then $g \leq f$, and it is easy to see by the monotone convergence theorem that $g$ is summable and $\int_{A} g d \mu(x)=a$. Let $\varepsilon>0$, and suppose that $\mu^{*}(B)<\mu(A)$, where $B=\{x \in A: f(x)<g(x)+\varepsilon\}$. Then there is a measurable set $C \subset A$ of positive measure such that $f \geq g+\varepsilon$ on $C$. Putting $h=g+\varepsilon \cdot \chi_{C}$, we find $h \leq f$ and $\int_{A} h d \mu(x)>a$, which contradicts the definition of $a$.

Lemma 2. For every $f: A \rightarrow \overline{\mathbb{R}}$ and $g: A \rightarrow \overline{\mathbb{R}}$ we have

(1) $\int_{=}(f+g) d \mu(x) \geq \underline{=}_{A} f d \mu(x)+\underline{\underline{S}}_{A} g d \mu(x)$ and

(2) $\overline{\bar{J}}_{A}^{A}(f+g) d \mu(x) \leq \overline{\bar{\int}}_{A}^{A} f d \mu(x)+\overline{\bar{\int}}_{A}^{A} g d \mu(x)$,

whenever the right-hand sides make sense.

Proof. This follows directly from the definitions. 
Lemma 3. For every sequence of nonnegative functions $f_{n}: A \rightarrow[0, \infty]$ we have

$$
\bar{\int}_{A} \liminf _{n} f_{n} d \mu(x) \leq \liminf _{n} \bar{\int}_{A} f_{n} d \mu(x) .
$$

Proof. By the dual of Lemma 1 there are measurable functions $g_{n}: A \rightarrow[0, \infty]$ such that $f_{n} \leq g_{n}$ and $\bar{\int}_{A} f_{n} d \mu(x)=\int_{A} g_{n} d \mu(x)$ for every $n$ (if $\bar{\int}_{A} f_{n} d \mu(x)=\infty$, then we take $\left.g_{n} \equiv \infty\right)$. Then by Fatou's lemma we obtain

$$
\begin{aligned}
\int_{A} \liminf _{n} f_{n} d \mu(x) & \leq \int_{A} \liminf _{n} g_{n} d \mu(x)=\int_{A} \liminf _{n} g_{n} d \mu(x) \\
& \leq \liminf _{n} \int_{A} g_{n} d \mu(x)=\liminf _{n} \int_{A} f_{n} d \mu(x) .
\end{aligned}
$$

Theorem 4. For every probability space $(X, \Sigma, \mu)$ exactly one of the following statements is true.

(i) There is a measurable set of positive measure $A \subset X$ and a set $S \subset A \times I$ such that $\lambda\left(S_{x}\right)=1$ for every $x \in A$ and $\lambda^{*}\left(\left\{y \in I: \mu^{*}\left(S^{y}\right)<\mu(A)\right\}\right)>0$.

(ii) For every function $f: X \times I \rightarrow[0, \infty]$ we have

$$
\underline{\int}_{X}\left(\underline{\int f}(x, y) d y\right) d \mu(x) \leq \underline{\int}\left(\int_{X} f(x, y) d \mu(x)\right) d y .
$$

Proof. Suppose (i), and put $f=\chi_{S}$. Then the left-hand side of (9) equals $\mu(A)$. Let $C_{n}=\left\{y \in I: \mu^{*}\left(S^{y}\right)<\mu(A)-\frac{1}{n}\right\}$. If $n$ is large enough, then $\lambda^{*}\left(C_{n}\right)>0$, and thus the right-hand side of (9) is at most

$$
\left(1-\lambda^{*}\left(C_{n}\right)\right) \cdot \mu(A)+\lambda^{*}\left(C_{n}\right) \cdot(\mu(A)-(1 / n))<\mu(A),
$$

violating (ii).

Now suppose that (i) does not hold, and let $f: X \times I \rightarrow[0, \infty]$ be arbitrary. We prove (9). Suppose it is not true; that is,

$$
\underline{\int}_{X}\left(\underline{\int f}(x, y) d y\right) d \mu(x)>\underline{\int}\left(\int_{X} f(x, y) d \mu(x)\right) d y .
$$

We shall construct two sets, $A \subset X$ and $S \subset A \times I$, satisfying the conditions of (i). Since we assumed that (i) is false, this will complete the proof. We separate this part of the argument in the next lemma stating slightly more than what we actually need.

Lemma 5. Let $f: X \times I \rightarrow[0, \infty]$ and suppose that

$$
\underline{\int}_{X}\left(\underline{\int} f(x, y) d y\right) d \mu(x)>K \cdot \underline{\int}\left(\int_{X} f(x, y) d \mu(x)\right) d y,
$$

where $K>1$. Then there is a measurable set of positive measure $A \subset X$ and a set $S \subset A \times I$ such that $\lambda\left(S_{x}\right)=1$ for every $x \in A$ and $\lambda^{*}\left(\left\{y \in I: \mu^{*}\left(S^{y}\right)<\right.\right.$ $\mu(A) / K\})>0$.

Proof. Let $M$ denote the right-hand side of (10), and for each $x \in X$ put $g(x)=$ $\underline{\int f}(x, y) d y$. Since $\underline{\int}_{X} g d \mu(x)>K \cdot M$, there is a summable function $h: X \rightarrow \overline{\mathbb{R}}$ such that $0 \leq h(x) \leq g(x)$ for every $x \in X$, and $\int_{X} h d \mu(x)>K \cdot M$. Since $\int_{X} h d \mu(x)$ equals the supremum of the integrals of all step functions less than $h$, we may assume that $h$ itself is a step function; that is, $h=\sum_{i=1}^{N} c_{i} \chi_{A_{i}}$, where 
$c_{1}, \ldots, c_{N} \geq 0$ and $A_{1} \cup \ldots \cup A_{N}$ is a partition of $X$ into measurable sets. Note that

$$
\underline{\int f}(x, y) d y=g(x) \geq h(x)=c_{i} \text { for every } x \in A_{i}(i=1, \ldots, N) .
$$

It is easy to check, using the measurability of the sets $A_{i}$, that

$$
\sum_{i=1}^{N} \int_{A_{i}} f(x, y) d \mu(x)=\bar{\int}_{X} f(x, y) d \mu(x)
$$

for every $y$. Therefore, by Lemma 2 and by (11) we obtain

$$
\begin{aligned}
\sum_{i=1}^{N} K \cdot \int\left(\int_{A_{i}} f d \mu(x)\right) d y & \leq K \cdot \underline{\int}\left(\int_{X} f d \mu(x)\right) d y \\
& =K \cdot M<\int_{X} h d \mu(x)=\sum_{i=1}^{N} c_{i} \mu\left(A_{i}\right) .
\end{aligned}
$$

Hence there is an index $j$ such that

$$
K \cdot \underline{\int}\left(\bar{\int}_{A_{j}} f d \mu(x)\right) d y<c_{j} \mu\left(A_{j}\right)
$$

and since $f \geq 0$, we have $c_{j}>0$ and $\mu\left(A_{j}\right)>0$. Furthermore, by replacing $f$ by $f / c_{j}$, we may assume that $c_{j}=1$. Fix an $\varepsilon>0$ such that

$$
K \cdot \underline{\int}\left(\bar{\int}_{A_{j}} f(x, y) d \mu(x)\right) d y<(1-\varepsilon) \cdot \mu\left(A_{j}\right),
$$

and define $u: I \rightarrow \overline{\mathbb{R}}$ by $u(y)=\int_{A_{j}} f(x, y) d \mu(x)$. By Lemma 1 , there is a nonnegative summable function $k: I \rightarrow \overline{\mathbb{R}}$ such that $K \cdot \int k d y<(1-\varepsilon) \cdot \mu\left(A_{j}\right)$ and $\lambda^{*}(D)=1$, where $D=\left\{y \in I: u(y)<k(y)+\left(\varepsilon \cdot \mu\left(A_{j}\right)\right) / K\right\}$.

Now we define $F: A_{j} \times \Omega \rightarrow \overline{\mathbb{R}}$ by

$$
F(x, \bar{y})=\liminf _{n \rightarrow \infty} \frac{1}{n} \cdot \sum_{i=1}^{n} f\left(x, y_{i}\right)
$$

and put

$$
U=\left\{(x, \bar{y}) \in A_{j} \times \Omega: F(x, \bar{y}) \geq 1\right\} .
$$

For every $x \in A_{j}$ we have $\int f(x, y) d y \geq 1$ by (12) and thus, by the strong law of large numbers, $F(x, \bar{y}) \geq \overline{1}$ for a.e. $\bar{y} \in \Omega$. Therefore $\nu\left(U_{x}\right)=1$ (i.e. $U_{x}$ is measurable and of full measure in $\Omega$ ) for every $x \in A_{j}$. Applying the strong law of large numbers again, we find that the set

$$
E=\left\{\bar{y} \in \Omega: \lim _{n} \frac{1}{n} \cdot \sum_{i=1}^{n} k\left(y_{i}\right)=\int k d y<\frac{(1-\varepsilon) \cdot \mu\left(A_{j}\right)}{K}\right\}
$$

is a set of full measure in $\Omega$. Since $D$ is of full outer measure in $I$, it follows from [4, 254 L Theorem, p. 249] that $D^{\mathbb{N}}=D \times D \times \ldots$ and hence, $E \cap D^{\mathbb{N}}$ are of full 
outer measure in $\Omega$. If $\bar{y} \in E \cap D^{\mathbb{N}}$, then, by Lemmas 2 and 3 we have

$$
\begin{aligned}
\int_{A_{j}} F(x, \bar{y}) d \mu(x) & =\bar{\int}_{A_{j}}\left(\liminf _{n} \frac{1}{n} \cdot \sum_{i=1}^{n} f\left(x, y_{i}\right)\right) d \mu(x) \\
& \leq \liminf _{n} \bar{\int}\left(\frac{1}{n} \cdot \sum_{i=1}^{n} f\left(x, y_{i}\right)\right) d \mu(x) \\
& \leq \liminf _{n} \frac{1}{n} \cdot \sum_{i=1}^{n} \int_{A_{j}} f\left(x, y_{i}\right) d \mu(x) \\
& \leq \liminf _{n} \frac{1}{n} \cdot \sum_{i=1}^{n}\left(k\left(y_{i}\right)+(\varepsilon / K) \cdot \mu\left(A_{j}\right)\right) \\
& <(1-\varepsilon) \cdot\left(\mu\left(A_{j}\right) / K\right)+(\varepsilon / K) \cdot \mu\left(A_{j}\right)=\mu\left(A_{j}\right) / K
\end{aligned}
$$

Hence, for every $\bar{y} \in E \cap D^{\mathbb{N}}$

$$
\mu^{*}\left(U^{\bar{y}}\right)=\mu^{*}\left(\left\{x \in A_{j}: F(x, \bar{y}) \geq 1\right\}\right)<\mu\left(A_{j}\right) / K .
$$

That is, $U$ has the desired property but in the space $X \times \Omega$.

It now follows from the fact that $\Omega$ is isomorphic to $I$ that there is a set $S \subset X \times I$ such that $\lambda\left(S_{x}\right)=1$ for every $x \in A_{j}$, and $\mu^{*}\left(S^{y}\right)<\mu\left(A_{j}\right) / K$ for every $y$ belonging to a set of positive outer measure. This then completes the proof of Lemma 5 and hence the proof of Theorem 4 .

Theorem 6. The following statements are equivalent.

(i) There is no weak Sierpinski set.

(ii) For every nonnegative function $f:(I \times I) \rightarrow[0, \infty]$ we have

$$
\underline{\int}\left(\underline{\int} f(x, y) d y\right) d x \leq \underline{\int}\left(\int f(x, y) d x\right) d y .
$$

Proof. (ii) $\Longrightarrow$ (i): Obvious.

(i) $\Longrightarrow$ (ii): Suppose (ii) is not true. Then, by Theorem 4 there is a measurable set of positive measure $A \subset I$ and a set $U \subset A \times I$ such that $\lambda\left(U_{x}\right)=1$ for every $x \in A$ and $\lambda^{*}\left(\left\{y \in I: \lambda^{*}\left(U^{y}\right)<\lambda(A)\right\}\right)>0$. Since the measure space $A$ equipped with the normalized measure $\lambda / \lambda(A)$ is isomorphic to $(I, \lambda)$, we can find a set $T \subset I \times I$ such that $\lambda\left(T_{x}\right)=1$ for every $x \in I$ and $\lambda^{*}\left(\left\{y \in I: \lambda^{*}\left(T^{y}\right)<1\right\}\right)>0$.

Now we define $S=\{(x, y) \in I \times I:(x+r, y) \in T$ for every $r \in[-x, 1-x] \cap \mathbb{Q}\}$. Then $\lambda\left(S_{x}\right)=1$ for every $x \in I$. Moreover, if $\lambda^{*}\left(T^{y}\right)<1$, then $\lambda_{*}\left(I \backslash T^{y}\right)>0$ so that $I \backslash T^{y}$ contains a measurable set of positive measure. Hence for each such $y, \lambda\left(S^{y}\right)=0$. Thus $S$ is a weak Sierpiński set. This, however, contradicts our assumption, which completes the proof.

Corollary 7. It is consistent with ZFC that (2), (3), and (41) hold true for every bounded $f: I^{2} \rightarrow \mathbb{R}$.

Proof. Since the nonexistence of weak Sierpiński sets is consistent with ZFC (see 7]), it follows from the previous theorem that (3) holds consistently for every bounded $f$. Then, by duality, the same is true for (4), while (2) is a consequence of (3). 


\section{Consistent Density theorems}

Lemma 8. Let $(X, \Sigma, \mu)$ be a measure space. Then exactly one of the following two statements holds.

(i) There exists a set $F \subset X \times[0,1]$ such that $\lambda\left(F_{x}\right)=1$ for every $x \in X$, and $\lambda^{*}\left(\left\{y \in[0,1]: \mu\left(F^{y}\right)=0\right\}\right)>0$.

(ii) Whenever $H \subset X \times \mathbb{R}$ is such that $d_{*}\left(H_{x}, 0\right)=1$ for every $x \in X$, then there is a $\delta>0$ such that

$$
\underline{d}_{*}\left(\left\{y \in \mathbb{R}: \mu^{*}\left(H^{y}\right)>\delta\right\}, 0\right)>0 .
$$

Proof. Suppose $F \subset X \times[0,1]$ is such that $\lambda\left(F_{x}\right)=1$ for every $x \in X$, and $\lambda^{*}\left(\left\{y: \mu\left(F^{y}\right)=0\right\}\right)>0$. Let $y_{0} \in(0,1)$ be an outer density point of $\left\{y: \mu\left(F^{y}\right)=\right.$ $0\}$, and put $H=\left\{\left(x, y-y_{0}\right):(x, y) \in F\right\}$. It is clear that $H$ violates (ii), and thus (i) $\Longrightarrow \neg$ (ii).

Next suppose that statement (ii) is false. We prove that in this case (i) must hold. Let $H \subset X \times \mathbb{R}$ be such that $d_{*}\left(H_{x}, 0\right)=1$ for every $x \in X$, and let $\underline{d}_{*}\left(\left\{y \in \mathbb{R}: \mu^{*}\left(H^{y}\right)>1 / n\right\}, 0\right)=0$ for every $n=1,2, \ldots$ Then we can select $0<h_{n}<1 / n$ such that

$$
\lambda_{*}\left(\left\{y \in\left[0, h_{n}\right]: \mu^{*}\left(H^{y}\right)>1 / n\right\}\right)<h_{n} / 2^{n} .
$$

Put $\left.B_{n}=\left\{y \in[0,1]: \mu^{*}\left(H^{h_{n} y}\right) \leq 1 / n\right\}\right)$; then $\lambda^{*}\left(B_{n}\right)>1-2^{-n}$ for every $n$. Recall that $\Omega=I \times I \times \ldots$, and $\nu$ is the product measure on $\Omega$. If $B=B_{1} \times B_{2} \times \ldots$, then it follows from [4, 254 L Theorem, p. 249] that

$$
\nu^{*}(B)=\prod_{n=1}^{\infty}\left(1-\frac{1}{2^{n}}\right)>0 .
$$

We define $f_{n}: X \times I \rightarrow\{0,1\}$ by

$$
f_{n}(x, y)= \begin{cases}1 & \text { if }\left(x, h_{n} y\right) \in H \\ 0 & \text { otherwise }\end{cases}
$$

and define $\Phi: X \times \Omega \rightarrow[0,+\infty]$ by

$$
\Phi(x, \bar{y})=\liminf _{n \rightarrow \infty} \frac{1}{n} \cdot \sum_{i=1}^{n} f_{i}\left(x, y_{i}\right) .
$$

Fix $\bar{y} \in B$. Then for every $n \in \mathbb{N}, y_{n} \in B_{n}$ and thus

$$
\bar{\int} f_{n}\left(x, y_{n}\right) d \mu(x)=\mu^{*}\left(H^{h_{n} \cdot y_{n}}\right) \leq \frac{1}{n} .
$$

Therefore, by Lemmas 2 and 3 we have

$$
\begin{aligned}
\bar{\int} \Phi(x, \bar{y}) d \mu(x) & \leq \liminf _{n \rightarrow \infty} \frac{1}{n} \sum_{i=1}^{n} \bar{\int} f_{i}\left(x, y_{i}\right) d \mu(x) \\
& \leq \liminf _{n \rightarrow \infty} \frac{1}{n}\left(1+\frac{1}{2}+\ldots+\frac{1}{n}\right)=0 .
\end{aligned}
$$

Thus, for every $\bar{y} \in B$ we have $\Phi(x, \bar{y})=0$ for $\mu$-a.e. $x \in X$. 
On the other hand, for every $x \in X$ we have $\Phi(x, \bar{y})=1$ for $\nu$-a.e. $\bar{y} \in \Omega$. Indeed, from $d_{*}\left(H_{x}, 0\right)=1$ it follows that



Thus by the strong law of large numbers we obtain that

$$
\lim _{n \rightarrow \infty} \frac{f_{1}\left(x, y_{1}\right)+\ldots+f_{n}\left(x, y_{n}\right)}{n}=1
$$

for $\nu$-a.e. $\bar{y} \in \Omega$; that is, $\Phi(x, \bar{y})=1$ for $\nu$-a.e. $\bar{y}$. Now we put $F=\{(x, \bar{y}) \in$ $X \times \Omega: \Phi(x, \bar{y})=1\}$. Then $\nu\left(\Omega \backslash F_{x}\right)=0$ for every $x \in X$, and $\mu^{*}\left(F^{\bar{y}}\right)=0$ for every $\bar{y} \in B$. Since $\nu^{*}(B)>0$, we obtain the statement of (i) with $\Omega$ in place of $I$. As the measure spaces $(\Omega, \nu)$ and $(I, \lambda)$ are isomorphic, we find that (i) holds, which completes the proof.

Theorem 9. Suppose that there is no weak Sierpiniski set. Then, whenever $H \subset$ $I \times \mathbb{R}$ is such that $d_{*}\left(H_{x}, 0\right)=1$ for every $x \in I$, we have

$$
d_{*}\left(\left\{y: \lambda^{*}\left(H^{y}\right)>1-\delta\right\}, 0\right)=1
$$

for every $\delta>0$.

Proof. Let $H$ be as in the theorem, and let $\delta>0$ be arbitrary. Suppose (14) is false. Then there is an $\varepsilon>0$ and there is a sequence $h_{n}>0$ such that $h_{n} \rightarrow 0$ and

$$
\lambda_{*}\left(\left\{y \in\left[0, h_{n}\right]: \lambda^{*}\left(H^{y}\right)>1-\delta\right\}\right)<(1-\varepsilon) \cdot h_{n}
$$

for every $n$. Put $B_{n}=\left\{y \in I: \lambda^{*}\left(H^{h_{n} y}\right) \leq 1-\delta\right\}$; then $\lambda^{*}\left(B_{n}\right) \geq \varepsilon$ for every $n$. We define the functions $f_{n}$ and $\Phi$ as in the proof of Lemma 8 (with $I$ in place of $X)$. Then as in the proof of Lemma 8 , it can be shown that for every $x \in I$, we have $\Phi(x, \bar{y})=1$ for $\nu$-a.e. $\bar{y} \in \Omega$. If there is no weak Sierpiński set, then, by Theorem 6, we have

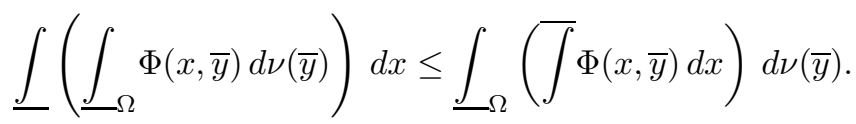

Since $\Phi(x, \bar{y})=1$ for $\nu$-a.e. $\bar{y} \in \Omega$, the left-hand side of (15) equals 1 . Then the right-hand side also equals 1 , and thus we have $\bar{\int} \Phi(x, \bar{y}) d x=1$ for $\nu$-a.e. $\bar{y}$. Then, by Lemma 3, we obtain

$$
1=\bar{\int} \Phi(x, \bar{y}) d x \leq \liminf _{n \rightarrow \infty} \frac{1}{n} \sum_{i=1}^{n} \bar{\int} f_{i}\left(x, y_{i}\right) d x=\liminf _{n \rightarrow \infty} \frac{1}{n} \sum_{i=1}^{n} \lambda^{*}\left(H^{h_{i} y_{i}}\right)
$$

for $\nu$-a.e. $\bar{y}$. Now we define $b_{i}=\chi_{B_{i}}$, and set

$$
D=\left\{\bar{y} \in \Omega: \limsup _{n \rightarrow \infty} \frac{1}{n} \sum_{i=1}^{n} b_{i}\left(y_{i}\right) \geq \varepsilon\right\} .
$$

We claim that $\nu^{*}(D)=1$. If the sets $B_{i}$ were measurable, then this would follow from the strong law of large numbers. In the general case we can argue as follows. Let $M_{i}$ be a measurable hull of $B_{i}$, and consider the measure spaces $\left(X_{i}, \Sigma_{i}, \lambda\right)$, where $X_{i}=\left(I \backslash M_{i}\right) \cup B_{i}$, and $\Sigma_{i}$ is the $\sigma$-algebra of all sets of the form $X_{i} \cap A$, where $A$ is Lebesgue measurable. Then $B_{i} \in \Sigma_{i}$. Let $\Omega_{1}=X_{1} \times X_{2} \times \ldots$, and let $\nu_{1}$ denote the product measure on $\Omega_{1}$. It is easy to check that $\nu^{*}(A)=\nu_{1}(A)$ for 
every $\nu_{1}$-measurable $A \subset \Omega_{1}$. Since, by the strong law of large numbers, $\nu_{1}(D)=1$, it follows that $\nu^{*}(D)=1$.

But if $y \in B_{i}$, then $\lambda^{*}\left(H^{h_{i} y}\right) \leq 1-\delta$, and therefore

$$
\lambda^{*}\left(H^{h_{i} y}\right) \leq 1-\delta \cdot b_{i}(y)
$$

for every $y \in I$ and $i=1,2, \ldots$ Let $\bar{y} \in D$ be arbitrary. By (18) and (17) we have

$$
\liminf _{n \rightarrow \infty} \frac{1}{n} \sum_{i=1}^{n} \lambda^{*}\left(H^{h_{i} y_{i}}\right) \leq\left[1-\delta \cdot \limsup _{n \rightarrow \infty} \frac{1}{n} \sum_{i=1}^{n} b_{i}\left(y_{i}\right)\right] \leq 1-\delta \cdot \varepsilon .
$$

Since $\nu^{*}(D)=1$, this contradicts the fact that (16) holds for $\nu$-a.e. $\bar{y}$, which completes the proof.

Theorem 10. Suppose $\operatorname{shr}(\mathcal{N})<\operatorname{cov}(\mathcal{N})$. Let $E_{1}, E_{2} \subset \mathbb{R}$ be measurable sets of positive measure, and let $H \subset E_{1} \times E_{2}$ be such that $\lambda_{*}\left(H_{x}\right)>0$ for every $x \in E_{1}$. Then there is a $y \in E_{2}$ and there is a $\delta>0$ such that

$$
\underline{d}_{*}\left(\left\{z \in E_{2}: \lambda^{*}\left(H^{y} \cap H^{z}\right)>\delta\right\}, y\right)>0 .
$$

Proof. Let $E_{1}, E_{2}, H$ be as in the theorem. We may assume, by deleting a set of measure zero from $E_{2}$, that $E_{2}$ is nonempty and $d$-open; i.e. that every point of $E_{2}$ is a density point of $E_{2}$. Also, by taking a suitable subset of $H$, we may assume that $H_{x}$ is nonempty and $d$-open for every $x \in E_{1}$. Suppose that the conclusion of the theorem is not true; that is,

$$
\underline{d}_{*}\left(\left\{z \in E_{2}: \lambda^{*}\left(H^{y} \cap H^{z}\right)>\delta\right\}, y\right)=0
$$

for every $y \in E_{2}$ and $\delta>0$.

First we show that, for every $y \in E_{2}, H^{y}$ can be covered by $\operatorname{shr}(\mathcal{N})$-many null sets. This is obvious if $\lambda^{*}\left(H^{y}\right)=0$. Let $y \in E_{2}$ be fixed such that $\lambda^{*}\left(H^{y}\right)>0$, and put $X=H^{y}$. Since $H_{x}$ is $d$-open for every $x \in X$, we have $d\left(H_{x}, y\right)=1$ for every $x \in X$.

We apply Lemma 8 to the measure space $\left(X, \lambda^{*} \mid X\right)$ (the restriction of $\lambda^{*}$ to the subsets of $X)$ and for the set $\{(x, z): x \in X,(x, z+y) \in H\}$. Since, by (20), the statement (ii) of Lemma 8 is not true, we obtain a set $F \subset X \times I$ such that $\lambda\left(F_{x}\right)=1$ for every $x \in X$, and

$$
\lambda^{*}\left(\left\{z \in I: \lambda^{*}\left(F^{z}\right)=0\right\}\right)>0 .
$$

It is the existence of such a set that implies that $X$ can be covered by $\operatorname{shr}(\mathcal{N})$ many null sets. To see this, let $A \subset\left\{z \in I: \lambda^{*}\left(F^{z}\right)=0\right\}$ be a set such that $|A| \leq \operatorname{shr}(\mathcal{N})$ and $\lambda^{*}(A)>0$. Then we claim that $X=\bigcup_{z \in A} F^{z}$. Indeed, if $x \in X$, then $\lambda\left(F_{x}\right)=1$ and since $\lambda^{*}(A)>0, F_{x} \cap A \neq \emptyset$. But if $z \in F_{x} \cap A$, then $x \in F^{z}$ so that $x \in \bigcup_{z \in A} F^{z}$.

Summing up, assuming the theorem is false, we have proved that for every $y \in$ $E_{2}, H^{y}$ can be covered by $\operatorname{shr}(\mathcal{N})$-many null sets. Now, define

$$
K=\{(x, y+r):(x, y) \in H, r \in \mathbb{Q}\} .
$$

Then $K_{x}$ is a measurable set and of full measure for every $x \in E_{1}$. Moreover, for every $y \in \mathbb{R}, K^{y}$ is the union of countably many horizontal sections of $H$ and so for every $y \in \mathbb{R}, K^{y}$ can be covered by $\operatorname{shr}(\mathcal{N})$-many null sets. Let $B \subset \mathbb{R}$ be a set such that $|B| \leq \operatorname{shr}(\mathcal{N})$ and $\lambda^{*}(B)>0$. Then $E_{1}=\bigcup_{z \in B} K^{z}$, and since each $K^{z}$ can be covered by $\operatorname{shr}(\mathcal{N})$-many null sets, the same is true for $E_{1}$. It follows that $\mathbb{R}$ can be covered by shr $(\mathcal{N})$ many null sets, since we can cover $\mathbb{R}$ by a null set and by 
countably many translated copies of $E_{1}$. Thus we obtain $\operatorname{cov}(\mathcal{N}) \leq \operatorname{shr}(\mathcal{N})$, which contradicts our assumption.

\section{Symmetrically apPROXimately CONTINUOUS FUnCTIONS}

Let $S \subset \mathbb{R}^{2}$ be arbitrary. We say that the points $x, y \in \mathbb{R}$ can be joined by a $k$-chain using the centers $p_{1}, \ldots, p_{k}$, if there are points $x=x_{0}, x_{1}, \ldots, x_{k}=y$ such that $x_{i-1}+x_{i}=2 p_{i}$ and $\left(p_{i}, x_{i-1}\right) \in S$ for every $i=1, \ldots, k$.

Theorem 11. Suppose $\operatorname{shr}(\mathcal{N})<\operatorname{cov}(\mathcal{N})$. Let $E \subset \mathbb{R}$ be a measurable set of positive measure, and let $S \subset \mathbb{R}^{2}$ be a set such that $d_{*}\left(S_{x}, x\right)=1$ for a.e. $x \in E$. Then there is a point $x_{0} \in E$ and there is a measurable set $F \subset E$ of positive measure such that every point of $F$ can be joined to $x_{0}$ by a suitable 4-chain in $E$.

Proof. We may assume that $E$ is a nonempty $d$-open subset of the interval $\left[\frac{1}{4}, \frac{1}{2}\right]$. We may also suppose that $d_{*}\left(S_{x}, x\right)=1$ for a.e. $x \in \mathbb{R}$. Indeed, let $V$ denote the $d$-interior of $\mathbb{R} \backslash E$, and replace $S$ by the set $S^{\prime}=(S \cap(E \times \mathbb{R})) \cup(V \times V)$. Suppose we can prove that there is a point $x_{0} \in E$ and a measurable set $F \subset E$ of positive measure such that every point of $F$ can be joined to $x_{0}$ by a suitable 4-chain using $S^{\prime}$ instead of $S$. Then, in every chain consisting of elements of $E$, the centers must also belong to $E$ since for $p \notin E, S_{p}^{\prime} \cap E=V \cap E=\emptyset$. Therefore, if two points of $E$ can be connected by a chain in $E$ using the set $S^{\prime}$, then they also can be connected using $S$. Taking a suitable subset of $S$ we may also assume that $S$ has the following properties:

(i) for every $x$ the section $S_{x}$ is $d$-open;

(ii) for a.e. $x$ the section $S_{x}$ is nonempty;

(iii) for every $x$, if $S_{x} \neq \emptyset$, then $d_{*}\left(S_{x}, x\right)=1$;

(iv) if $x \in E$, then $S_{x} \subset E$ and if $x \notin E$, then $S_{x} \cap E=\emptyset$; and

(v) for every $x$, the section $S_{x}$ is symmetric about $x$; that is, $2 x-S_{x}=S_{x}$.

By applying Theorem 10 for the set $S \cap(E \times E)$ we obtain a point $x_{0} \in E$ and a $\delta>0$ such that

$$
\underline{d}_{*}\left(\left\{y \in E: \lambda^{*}\left(S^{x_{0}} \cap S^{y} \cap E\right)>\delta\right\}, x_{0}\right)>0 .
$$

Let $T=\{(x, y): x \in I,(x, y+x) \in S\}$. Then $T_{x}=S_{x}-x$, and thus $d_{*}\left(T_{x}, 0\right)=1$ for a.e. $x \in I$. Since $\operatorname{shr}(\mathcal{N})<\operatorname{cov}(\mathcal{N})$ implies that there is no weak Sierpiński set, we may apply Theorem 9 to obtain

$$
d_{*}\left(\left\{y \in \mathbb{R}: \lambda^{*}\left(T^{y}\right)>1-\delta\right\}, 0\right)=1 .
$$

It now follows from (21) and (22) that there is a measurable set $F$ of positive measure such that

$$
\begin{aligned}
F \subset E \cap\left\{y \in\left[x_{0}-\delta, x_{0}+\delta\right]:\right. & \\
& \left.\lambda^{*}\left(S^{x_{0}} \cap S^{y} \cap E\right)>\delta, \lambda^{*}\left(T^{\left(y-x_{0}\right) / 2}\right)>1-\delta\right\} .
\end{aligned}
$$

(In fact, we can find such a measurable set $F$ with $\underline{d}_{*}\left(F, x_{0}\right)>0$.) We complete the proof by showing that every point of $F$ can be joined to $x_{0}$ by a 4 -chain.

Let $y \in F$ be arbitrary. By (23), we have $\lambda^{*}\left(S^{x_{0}} \cap S^{y} \cap E\right)>\delta$. Let $D$ denote the set of outer density points of $S^{x_{0}} \cap S^{y} \cap E$. Then $D$ is measurable and $\lambda(D)>\delta$. As $E \subset\left[\frac{1}{4}, \frac{1}{2}\right]$, it follows that $x_{0} \in\left[\frac{1}{4}, \frac{1}{2}\right]$ and both $S^{x_{0}} \cap S^{y} \cap E \subset\left[\frac{1}{4}, \frac{1}{2}\right]$ and $D \subset\left[\frac{1}{4}, \frac{1}{2}\right]$. Hence, $2 D-x_{0} \subset I$ is measurable and $\lambda\left(2 D-x_{0}\right)>2 \delta$. Since $T^{\left(y-x_{0}\right) / 2} \subset I, \lambda^{*}\left(T^{\left(y-x_{0}\right) / 2}\right)>1-\delta$ and $\left|y-x_{0}\right| \leq \delta$, it follows that 
$\lambda^{*}\left(\left[2 D-x_{0}\right] \cap\left[T^{\left(y-x_{0}\right) / 2}+\left(y-x_{0}\right) / 2\right]\right)>0$. Fix an element $q \in\left[2 D-x_{0}\right] \cap$ $\left.\left[T^{\left(y-x_{0}\right) / 2}+\left(y-x_{0}\right) / 2\right]\right)$ such that $S_{q} \neq \emptyset$. We claim that the set

$$
U=\left(S^{x_{0}} \cap S^{y}\right) \cap \frac{1}{2}\left[x_{0}+S_{q}\right] \cap \frac{1}{2}\left[\left(2 q+x_{0}\right)-S_{q-\left(y-x_{0}\right) / 2}\right]
$$

is nonempty. Indeed, as $q \in 2 D-x_{0}$, then $\left(q+x_{0}\right) / 2$ is an outer density point of $S^{x_{0}} \cap S^{y}$. Since $q$ is a density point of $S_{q}$, it follows that $\left(q+x_{0}\right) / 2$ is a density point of $\frac{1}{2}\left[x_{0}+S_{q}\right]$. The condition $q \in T^{\left(y-x_{0}\right) / 2}+\left(y-x_{0}\right) / 2$ implies

$$
\left(q-\left(y-x_{0}\right) / 2,\left(y-x_{0}\right) / 2\right) \in T \quad \text { and } \quad\left(q-\left(y-x_{0}\right) / 2, q\right) \in S ;
$$

that is, $q \in S_{q-\left(y-x_{0}\right) / 2}$. Therefore $q$ is a density point of $S_{q-\left(y-x_{0}\right) / 2}$, and thus $\left(q+x_{0}\right) / 2$ is a density point of $\frac{1}{2}\left[\left(2 q+x_{0}\right)-S_{q-\left(y-x_{0}\right) / 2}\right]$. Therefore, $\left(q+x_{0}\right) / 2$ is an outer density point of $U$, and thus $U \neq \emptyset$. Let $p \in U$.

We claim that $x_{0}, 2 p-x_{0}, 2 q-2 p+x_{0}, 2 p-y, y$ is a 4-chain, using the centers $p, q, q-\left(y-x_{0}\right) / 2$, and $p$. All that we have to check is that the relations

$$
x_{0} \in S_{p}, 2 p-x_{0} \in S_{q}, 2 q-2 p+x_{0} \in S_{q-\left(y-x_{0}\right) / 2}, \text { and } 2 p-y \in S_{p}
$$

hold. The first three of these relations follow from $p \in U$ (note that if $p \in S^{x_{0}}$, then $x_{0} \in S_{p}$ ). To see that $2 p-y \in S_{p}$ it is enough to check that $y \in S_{p}$ since $S_{p}$ is symmetric about $p$. But, $p \in S^{y}$ so $y \in S_{p}$ and this completes the proof.

Theorem 12. Suppose $\operatorname{shr}(\mathcal{N})<\operatorname{cov}(\mathcal{N})$. If $E \subset \mathbb{R}$ is measurable and $f: E \rightarrow \mathbb{R}$ is symmetrically approximatively continuous at a.e. point of $E$, then $f$ is measurable.

Proof. It is enough to show that for every $\varepsilon>0$ there is a partition $E=\bigcup_{n=1}^{\infty} E_{n}$ such that each $E_{n}$ is measurable, and for every $n$, the oscillation of $f \mid E_{n}$ is at most $\varepsilon$. By using a standard exhaustion argument, it is enough to prove that there exists a measurable set $F \subset E$ of positive measure such that the oscillation of $f \mid F$ is at most $\varepsilon$.

Since $f$ is symmetrically approximatively continuous at a.e. point of $E$, for a.e. $x \in E$ there is a set $S_{x}$ such that $d_{*}\left(S_{x}, x\right)=1$, and $|f(x+h)-f(x-h)|<\varepsilon / 8$ whenever $x+h, x-h \in S_{x}$. Put $S=\left\{(x, y): y \in S_{x}\right\}$. By Theorem 11] there is a point $x_{0} \in E$ and a measurable set $F \subset E$ of positive measure such that every point of $F$ can be joined to $x_{0}$ by a suitable 4 -chain. It is clear that if the points $x_{0}$ and $y$ can be joined by a 4-chain, then $\left|f\left(x_{0}\right)-f(y)\right|<\varepsilon / 2$. Therefore $\left|f\left(x_{0}\right)-f(y)\right|<\varepsilon / 2$ holds for every $y \in F$, and thus the oscillation of $f \mid F$ is at most $\varepsilon$.

\section{REFERENCES}

[1] K. Ciesielski, Generalized continuities. In: Encyclopedia of General Topology (J.I. Nagata, J.E. Vaughan, and K.P. Hart, eds.), Elsevier, to appear.

[2] C. Freiling, Axioms of symmetry: throwing darts at the real number line, J. Symb. Logic 51 (1986), 190-200. MR0830085 (87f:03148)

[3] C. Freiling, A converse to a theorem of Sierpiński on almost symmetric sets, Real Anal. Exchange 15 (2) (1989-90), 760-767. MR1059437 (91d:26009)

[4] D. H. Fremlin, Measure Theory, Vol. 2. Torres Fremlin, Colchester, 2001.

[5] P. D. Humke and M. Laczkovich, Parametric semicontinuity implies continuity, Real Anal. Exchange 17 (2) (1991-92), 668-680. MR.1171407 (93g:26004)

[6] M. Kada and Y. Yuasa, Cardinal invariants about shrinkability of unbounded sets, Topology Appl. 74 (1996), 215-223. MR1425940|(97j:03092)

[7] M. Laczkovich, Two constructions of Sierpiński and some cardinal invariants of ideals, Real Anal. Exchange 24 (2) (1998-99), 663-676. MR1704742 (2000f:03148) 
[8] M. Laczkovich and A. W. Miller, Measurability of functions with approximately continuous vertical sections and measurable horizontal sections, Colloq. Math. 69(2) (1995), 299-308. MR 1358935 (96k:28005)

[9] I. N. Pesin, The measurability of symmetrically continuous functions (Russian), Teor. Funkciǔ. Funkcional. Anal. i Priložen Vyp. 5 (1967), 99-101. MF0227331 (37:2916)

[10] D. Preiss, A note on symmetrically continuous functions, Časopis Pěst. Mat. 96 (1971), 262-264, 300. MR0306411 (46:5537)

[11] W. Sierpiński, Sur une fonction non mesurable, partout presque symétrique, Acta Litt. Scient. (Szeged) 8 (1936), 1-6. Reprinted in: Oeuvres Choisies, PWN - Editions Scientifiques de Pologne, Warszawa 1974. Vol. III, pp. 277-281. MR0414302 (54:2405)

[12] B. S. Thomson, Symmetric Properties of Real Functions. Monographs and Textbooks in Pure and Applied Mathematics, 183. Marcel Dekker, Inc., New York, 1994. MR1289417 (95m:26002)

[13] J. Uher, Symmetric semicontinuity implies continuity, Trans. Amer. Math. Soc. 293 (1986), no. 1, 421-429. MF0814930 (87b:26005)

Department of Mathematics, Washington and Lee University, Lexington, Virginia 24450 - and - Department of Mathematics, St. Olaf College, Northfield, Minnesota 55057

E-mail address: humke@stolaf.edu

Department of Analysis, Eötvös Loránd University, Budapest, PÁzmány Péter sétány 1/C, 1117 Hungary - and - Department of Mathematics, University College London, Gower Street, London, WC1E 6BT, England

E-mail address: laczk@cs.elte.hu 